\title{
THE SCOPE OF THE ENERGY METHOD ${ }^{1}$
}

\author{
BY PETER D. LAX
}

Communicated by Lipman Bers, September 11, 1959

In this paper the energy method is applied to show the stability of a certain class of difference approximations to linear hyperbolic partial differential equations with variable coefficients. We shall consider first order hyperbolic systems:

$$
u_{t}=A u_{x},
$$

$u$ a vector, $A$ a coefficient matrix with real and distinct eigenvalues. We approximate this by explicit, one level difference schemes, i.e., of the form

$$
v_{k}=\sum_{-N}^{N} c_{j} u_{k-j}
$$

where $u_{j}$ and $v_{j}$ denote the values of the approximate solution at position $j \Delta$ and at times $t$ and $t+\Delta$ respectively. We have chosen here for simplicity the same value $\Delta$ for both time and space increments.

The coefficients $c_{j}$ depend on the index $k$; we assume that this dependence is Lipschitz continuous, i.e., that $c(k+1)-c(k)=O(\Delta)$.

Since the differential equation (1) is homogeneous, $u=$ const. is a solution. We assume, as part of the consistency of (1) and (2), that $u=$ const. also is a solution of (2), i.e., that

$$
\sum c_{j}=I \text {. }
$$

A difference scheme is called stable in the $L_{2}$-norm if the $L_{2}$-norm of any solution at any given time $t=T$ remains uniformly bounded, as $\Delta$ tends to zero, by a constant multiple of its $L_{2}$-norm at $t=0$. It is well known, see [4], that if (2) is formally consistent with (1) then solutions of (2) approach solutions of (1) as $\Delta$ tends to zero if and only if (2) is stable.

If the coefficients of (2) are constant, then the question of stability can be easily settled by Fourier analysis.

Put

$$
V(\theta)=\sum v_{k} e^{i k \theta}, \quad U(\theta)=\sum u_{j} e^{i j \theta}
$$

then it follows from (2) that

1 The work presented in this paper is supported by the AEC Computing and Applied Mathematics Center, Institute of Mathematical Sciences, New York University, under Contract AT (30-1)-1480 with the U. S. Atomic Energy Commission. 


$$
V(\theta)=C(\theta) U(\theta)
$$

where

$$
C(\theta)=\sum c_{j} e^{i j \theta} .
$$

Restricting this discussion at the present to the scalar case, we observe that in terms of $C(\theta)$, (3) can be rewritten as

$$
C(\theta)=1 \text {. }
$$

One obtains, as necessary and sufficient for stability, the condition of von Neumann:

For all real $\theta$

$$
|C(\theta)| \leqq 1 .
$$

It is easy to show that, even when the coefficients vary, it is necessary for stability that von Neumann's condition be satisfied at each point. It is a long standing conjecture, supported by much numerical evidence, that this is sufficient as well; here we present a proof of this conjecture. A further generalization to the matrix case is indicated below. The various interesting special cases which have been treated before are listed at the end. The corresponding problem for parabolic equations has been solved by John [2].

THEOREM. If the coefficients in the difference scheme vary Lipschitz continuously and have Property (A) below, and if von Neumann's condition is satisfied at every point then the scheme is stable.

Property (A). As $k$ varies, the multiplicity of the roots of $1-|C(\theta)|^{2}$ change at most by 2.

Denote the array of the coefficients $c_{-N}, \cdots, c_{N}$ by $c$. The proof of the theorem is based on the following:

Lemma. Suppose that the trigonometric polynomial $C(\theta)$ of degree $N$ satisfies conditions (5) and (6); then there exists a $2 N+1$ by $2 N+1$ matrix $Q$ with the following properties:

$(\alpha) Q$ is hermitean and nonnegative,

$(\beta)$ The sum of the elements of $Q$ along the main diagonal is 1 , along any subdiagonal is zero:

$$
\sum q_{r-k, 8-k}=\delta_{r, s}
$$

$(\gamma)$ The row sums of $Q$ give the coefficients of $C(\theta)$, i.e.,

$$
Q e=c,
$$

e being the vector all whose components are 1.

PRoof. Introduce 


$$
K(\theta, \phi)=\sum q_{l m} e^{i l \theta-i m \phi} .
$$

Properties $(\alpha),(\beta),(\gamma)$ of $Q$ are equivalent to these of $K$ :

$\left(\alpha^{\prime}\right) K(\theta, \phi)$ is the kernel of a hermitean nonnegative operator.

$\left(\beta^{\prime}\right) K(\theta, \theta)=1$.

$\left(\gamma^{\prime}\right) K(\theta, \theta)=C(\theta)$.

According to (6), $1-|C(\theta)|^{2}$ is a nonnegative trigonometric polynomial of degree $2 N$. Therefore by the well known Fejér-Riesz theorem it is the square of the absolute value of a trigonometric polynomial $D(\theta)$ of degree $N$. It is now easily seen, using (5), that

$$
K(\theta, \phi)=C(\theta) \bar{C}(\phi)+D(\theta) \bar{D}(\phi)
$$

has properties $\left(\alpha^{\prime}\right),\left(\beta^{\prime}\right),\left(\gamma^{\prime}\right)$. This proves the lemma.

It is not difficult to show that under the stated conditions $Q$ can be chosen to depend Lipschitz continuously on $k$.

In obvious vector notation and with the representation $(\gamma)$ for $c$, (2) can be written as

$$
v_{k}=\left(c, u_{k}\right)=\left(Q e, u_{k}\right) .
$$

Since by $(\alpha) Q$ is nonnegative, we can apply Schwarz's inequalty, obtaining

$$
v_{k}^{2} \leqq(Q e, e)\left(Q u_{k}, u_{k}\right) .
$$

$(Q e, e)=1$ by $(\beta)$; summing with respect to $k$ we get

$$
\sum v_{k}^{2} \leqq \sum q_{l m}(k) u_{k+l} u_{k+m} .
$$

It follows from $(\beta)$ and the Lipschitz continuity of $Q$ that

$$
\sum_{k} q_{r-k, 8-k}^{(k)}=\delta_{r, s}+O(\Delta) .
$$

So it follows from (7) that

$$
\sum v_{k}^{2} \leqq\{1+O(\Delta)\} \sum u_{k}^{2} .
$$

From this it follows that after $n$ time steps the $L_{2}$-norm is increased at most by a factor

$$
\{1+O(\Delta)\}^{n}=\exp O(n \Delta)=\exp O(T) .
$$

This proves the stability of the scheme.

In the matrix case the necessary and nearly sufficient condition for stability of schemes with constant coefficients is that the spectral radius of $C(\theta)$ is $\leqq 1$ for all real $\theta$. It is conjectured that this is suffi- 
cient for stability even when the coefficients vary. If we strengthen this condition (see [5]) to

$$
C^{*}(\theta) C(\theta) \leqq I \quad \text { for } \theta \text { real, }
$$

then the present proof applies if we make use of Rosenblatt's generalization to matrices of the Fejér-Riesz theorem.

If condition $\left(6^{\prime}\right)$ is not satisfied then in order to apply the method of this note we have to replace the norm $\sum u_{k}^{2}$ by a sum of squares of appropriate linear combinations of $u_{k}$. In this case we can no longer require that $K$ be a trigonometric polynomial; the same holds for the case of more than one space variables, where in addition we have to represent $1-|C|^{2}$ as a sum of squares rather than a single square.

The stability of schemes with variable coefficients which are symmetric and nonnegative (in which case condition (6) is satisfied) has been proved by Friedrichs for any number of variables, see [1], also [4]. This does not cover many schemes of practical interest, such as the three-term third order scheme

$$
v_{k}=\frac{A^{2}-A}{2} u_{k-1}+\left(I-A^{2}\right) u_{k}+\frac{A^{2}+A}{2} u_{k+1} .
$$

This scheme satisfies $\left(6^{\prime}\right)$ if the Courant-Friedrichs-Lewy condition $-I \leqq A \leqq I$, is fulfilled but its coefficients are not $\geqq 0$.

Interesting energy inequalities for certain implicit schemes for the second order wave equation have been derived by $M$. Lees in a paper to appear in Trans. Amer. Math. Soc. Energy identities for an implicit difference scheme applicable to first order systems have been found by B. Wendroff in his N.Y.U. dissertation. Further interesting uses of the energy method are given in Kreiss, [3].

\section{BIBLIOGRAPHY}

1. K. O. Friedrichs, Symmetric hyperbolic linear differential equations, Comm. Pure Appl. Math. vol. 7 (1954) p. 345.

2. F. John, On the integration of parabolic equations by difference methods, Comm. Pure Appl. Math. vol. 5 (1952) p. 155.

3. H. O. Kreiss, Über die Lösung des Cauchyproblems für lineare partielle Differenzialgleichungen mit Hilfe von Differenzengleichungen, Acta Math. vol. 101 (1959) pp. 179-199.

4. P. D. Lax and R. D. Richtmyer, Survey of stability of linear finite difference equations, Comm. Pure Appl. Math. vol. 8 (1955) pp. 267-293.

5. R. D. Richtmyer, Difference methods for initial-value problems, Interscience Tracts in Pure and Applied Mathematics, vol. 4, 1957.

New York UnIVERSITY 